\title{
Bond tilting and sliding friction in a model of cell adhesion
}

\author{
By S. Reboux, G. Richardson and O. E. Jensen* \\ School of Mathematical Sciences, University of Nottingham, \\ University Park, Nottingham NG7 2RD, UK
}

\begin{abstract}
As a simple theoretical model of a cell adhering to a biological interface, we consider a rigid cylinder moving in a viscous shear flow near a wall. Adhesion forces arise through intermolecular bonds between receptors on the cell and their ligands on the wall, which form flexible tethers that can stretch and tilt as the base of the cell moves past the wall; binding kinetics is assumed to follow a standard model for slip bonds. By introducing a finite resistance to bond tilting, we use our model to explore the territory between previous theoretical models that allow for either zero or infinite resistance to bond rotation. A microscale calculation (for two parallel sliding plates) reveals a nonlinear force-speed relation arising from bond formation, tilting and breakage. Two distinct types of macroscale cell motion are then predicted: either bonds adhere strongly and the cell rolls (or tank treads) over the wall without slipping, or the cell moves near its freestream speed with bonds providing weak frictional resistance to sliding. The model predicts bistability between these two states, implying that at critical shear rates the system can switch abruptly between rolling and free sliding, and suggesting that sliding friction arising through bond tilting may play a significant dynamical role in some celladhesion applications.
\end{abstract}

Keywords: molecular friction; adhesion; bistability; cell rolling

\section{Introduction}

The adhesion of cells in suspension to nearby surfaces is a widespread and biologically significant process. Blood-borne cells, for example, stick to the endothelial lining of blood vessels during the immune response and cancer cell metastasis (Zhu 2000; Liotta 2001). Bacteria can bind to medical implants or host cells in the presence of an external flow during the course of an infection (Isberg \& Barnes 2002) and functionalized particles or microbubbles are candidates for targeted drug delivery (Moore \& Kuhl 2006; Rychak et al. 2006). Cell adhesion is commonly mediated by specific receptor-ligand interactions, which enable leucocytes, for example, to roll over the surface of post-capillary venules prior to targeting sites of inflammation (Springer 1990; Schmidtke \& Diamond 2000; McEver 2001). The recent identification of some of the key adhesion molecules (e.g. E-, L- and P-selectins and their ligands), and the

* Author for correspondence (oliver.jensen@nottingham.ac.uk). 
biomechanical characterization of the resulting intermolecular bonds (e.g. Alon et al. 1995; Tees et al. 2001), have stimulated the development of theoretical models of leucocyte rolling which have now reached considerable levels of sophistication (e.g. Krasik et al. 2006; Caputo et al. 2007). Multiscale models provide a powerful route to explore possible connections between physiological observations, such as the minimum shear threshold for rolling (Finger et al. 1996; Alon et al. 1997), and mechanochemical effects operating within individual intermolecular bonds (Hammer 2005). The adhesive properties of biological interfaces connected by multiple independent tethers are also presently inspiring the development of novel adhesives mimicking the remarkable properties of beetle and gecko feet (Gravish et al. in press; Varenberg \& Gorb 2007).

Adhesion of a cell to a biological surface necessarily involves numerous competing physical processes, some of which are specific to the cell type involved. These processes include cell and membrane deformation, flow of the suspending liquid and surrounding cells (Sun et al. 2003), receptor-ligand kinetics under time-varying forcing (Bell 1978; Dembo et al. 1988; Evans \& Ritchie 1997), deformation of surface structures such as bacterial pili (Andersson et al. 2006) and leucocyte microvilli (Shao et al. 1998; Ramachandran et al. 2004) and the complications of physical barriers such as endothelial glycocalyx (Zhao et al. 2001). Different theoretical frameworks can be used to model these different effects. The adhesive dynamics simulations of Hammer and co-workers, for example, have successfully captured the stochastic motion of rigid cells tethered by a small number of randomly distributed bonds that form and break under probabilistic rules (Hammer \& Apte 1992; Caputo et al. 2007). Intermolecular bonds are normally treated as simple Hookean springs, which may exhibit catch or slip behaviour (so that the dissociation rate, respectively, decreases or increases with applied force (e.g. Marshall et al. 2003)). Cell deformability is represented either within a largely computational framework (e.g. Dong \& Lei 2000; N'dri et al. 2003; Jadhav et al. 2005; Khismatullin \& Truskey 2005; Jin et al. 2007) or through more analytical approaches (Hodges \& Jensen 2002).

The aim of the present paper is to theoretically investigate the effects of relaxing a set of assumptions that underlies two families of existing models. In most theoretical models that represent bonds within a continuum framework, the bonds are treated as vertical springs that resist sideways displacement, preventing a cell membrane that is bound to a wall from sliding along it. This ensures that an adherent cell in a shear flow exhibits genuine tank-treading motion, with a peeling process taking place at the trailing edge of the contact region. This assumption underlies the models of Dembo et al. (1988), Dong \& Lei (2000), Hodges \& Jensen (2002), N'dri et al. (2003) and others. In contrast, theoretical models that treat bonds in a discrete framework (such as those based on adhesive dynamics) generally consider bonds as springs that form between individual contact points on the cell and substrate. The bonds are allowed to tilt freely as the contact points move past one another, enabling, in principle, some degree of sliding of the cell over the substrate.

In order to explore the territory between these two classes of models, we choose here to remain within the continuum deterministic limit (assuming that binding is mediated by a reasonably large number of bonds) but allow the bonds to tilt. To pass smoothly from the vertical bond limit to the case in which bonds can tilt freely, we assume that the bonds resist tilting via a biomechanical hinge of prescribed stiffness, 
while being subject to rotational diffusion. To our knowledge, bond resistance to tilting has yet to be characterized experimentally in the context of cell adhesion, although it is relevant in other biomimetic adhesives involving fields of oriented deformable binders (Varenberg \& Gorb 2007), and has motivated prior modelling of the adhesive properties of rotatable elastic nanofibres (Filippov \& Popov 2007). To isolate the effects of bond tilting from that of cell deformation, we represent the cell in two dimensions as a rigid circular cylinder moving in a low-Reynolds-number shear flow over a wall. We characterize the dynamic friction created by receptorligand bonds that continually form, stretch and break as the cylinder travels over the substrate through a combination of sliding and rolling motion. Some important features of sliding interactions between adhesive surfaces have already been identified by Chang \& Hammer (1999), who showed how sliding between a cell and its substrate increases the encounter rate but decreases the collision duration of receptor-ligand bonds forming between parallel interfaces, and by Yago et al. (2007), who related sliding motion, rotational molecular diffusion and Brownian motion of the cell to flow-augmented tethering of microspheres and neutrophils via L-selectin/PSGL-1 bonds. However, the sliding friction mediated by stretching and tilting bonds in this context has yet to be fully characterized theoretically.

Urbakh et al. (2004) recently suggested that the irregularity of leucocyte rolling over endothelium may be associated with the well-known stick-slip instability of dynamic friction that arises when a block attached to a spring is dragged over a rough surface. Models resolving individual intermolecular bonds between the block and surface (Filippov et al. 2004), or describing the motion of the block as a low-order dynamical system that incorporates a lumped representation of the dynamic frictional force (Drummond et al. 2003), have identified two necessary conditions for the stick-slip instability: a nonlinear relationship between the frictional force and the sliding velocity, and the dynamical degree of freedom (d.f.) associated with the block's inertia and the presence of the spring. While the latter is not an obvious feature of leucocyte rolling (casting doubt on the importance of stick-slip instabilities in this context), a nonlinear friction-velocity relation can certainly be anticipated in many cell-adhesion applications and is demonstrated in sliding friction of fibrillar adhesives (Varenberg \& Gorb 2007). We show here how bond tilting influences the friction-velocity relation between sliding surfaces and how this in turn can lead to novel threshold effects (i.e. hysteresis) in the relations between cell kinematics and imposed shear rate; such effects do not appear to have been described in previous cell-adhesion models that allow for bond tilting.

This paper is laid out as follows. We consider in detail the motion of a rigid cylinder moving in a two-dimensional flow above an adhesive wall. In $\$ 2$ we review the relevant hydrodynamics and present our model for reversible binding kinetics between sliding surfaces, accounting for bond tilting. This d.f. is described via a distribution function, while additional thermodynamic effects are built into forward and reverse reaction rates using a modified version of Dembo et al.'s (1988) popular model of binding kinetics under force. In §3a we derive a canonical friction-velocity relation between planar sliding surfaces and then we use a global force and torque balance in $\S 3 b$ to connect this to a macroscale model of the cylinder, leading to bistability in the cylinder's motion. In $\S 4$, we give order-of-magnitude estimates of the relevant dimensionless parameters for possible physiological applications and discuss the implications of our results. 


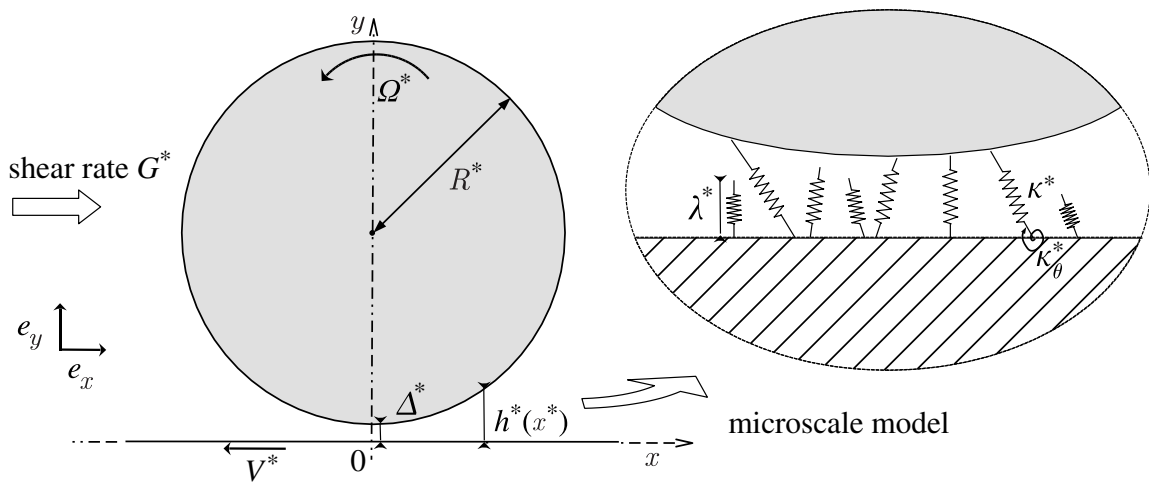

Figure 1. Motion of a rigid cylinder above an adhesive wall in a viscous fluid (as viewed in the frame of the cylinder).

\section{Model and methods}

We consider the motion of an infinite rigid horizontal cylinder of radius $R^{*}$ near a flat rigid horizontal wall. The cylinder moves over the wall in a shear flow of shear rate $G^{*}$ (figure 1). The fluid is assumed to be Newtonian with constant dynamic viscosity $\mu^{*}$, and the motion is governed by the Stokes equations with no-slip boundary conditions imposed on both the cylinder and the wall. The effects of gravity and inertia are neglected.

The wall is uniformly and densely coated with adhesive molecules (ligands) and the cylinder with receptors that are uniformly distributed with surface density $A_{\text {tot }}$. They interact via a reversible chemical process to form bound complexes, each of which behaves like a Hookean spring of unstressed length $\lambda^{*}$ and spring constant $\kappa$. Additionally, a torsional spring constant $\kappa_{\theta}$ is associated with the resistance of a molecular bond to being tilted away from its vertical equilibrium position.

As a result of the competition between the driving force of the shear flow, hydrodynamic drag and adhesion forces, the cylinder is assumed to move steadily parallel to the wall at a vertical distance $\Delta^{*}$ from it with speed $V^{*}$ in the $x$-direction and with anticlockwise angular speed $\Omega^{*}$ (see figure 1 ; note that we expect $\left.\Omega^{*}<0\right)$. We seek $\Delta^{*}, V^{*}$ and $\Omega^{*}$ in terms of the remaining parameters.

\section{(a) The macroscale problem: hydrodynamics}

Jeffrey \& Onishi (1981) derived the expression for the force and torque per unit width, $\boldsymbol{F}_{\mathrm{q}}^{*}$ and $\boldsymbol{T}_{\mathrm{q}}^{*}$, respectively, exerted on a cylinder moving slowly near a wall in a viscous quiescent fluid. At leading order in $\Delta^{*} / R^{*} \ll 1$, using lubrication theory, they obtained

$$
\boldsymbol{F}_{\mathrm{q}}^{*}=-\pi \mu^{*} \sqrt{8 R^{*} / \Delta^{*}} V^{*} \boldsymbol{e}_{x} \text { and } \boldsymbol{T}_{\mathrm{q}}^{*}=-\pi \mu^{*} \sqrt{8 R^{*} / \Delta^{*}} R^{* 2} \Omega^{*} \boldsymbol{e}_{z}
$$

where $\boldsymbol{e}_{x}$ and $\boldsymbol{e}_{y}$ are the horizontal and vertical unit vectors, respectively, and $\boldsymbol{e}_{z}=\boldsymbol{e}_{x} \times \boldsymbol{e}_{y}$ (figure 1). For a cylinder, $\boldsymbol{F}_{\mathrm{q}}^{*}$ is independent of $\Omega^{*}$ and $\boldsymbol{T}_{\mathrm{q}}^{*}$ is independent of $V^{*}$ to leading order.

Schubert (1967) computed the hydrodynamic force and torque per unit width, $\boldsymbol{F}_{\mathrm{s}}^{*}$ and $\boldsymbol{T}_{\mathrm{s}}^{*}$, respectively, exerted on a stationary cylinder resting on a wall in a 
shear flow, and found

$$
\boldsymbol{F}_{\mathrm{s}}^{*}=4 \pi \mu^{*} R^{*} G^{*} \boldsymbol{e}_{x} \text { and } \boldsymbol{T}_{\mathrm{s}}^{*}=-6 \pi \mu^{*} R^{* 2} G^{*} \boldsymbol{e}_{z} .
$$

At leading order as $\Delta^{*} / R^{*} \rightarrow 0$, the same expression applies to a stationary cylinder at a distance $\Delta^{*}$ from a wall in a shear flow. Briefly, this result arises from the fact that, with zero velocities at all the solid boundaries and zero pressure gradient at infinity, the flow near the narrowest part of the gap is very weak and the leading-order contribution to the force and torque comes only from the outer flow.

In view of the linearity of the Stokes equations, the total leading-order hydrodynamic force and torque per unit width exerted on a cylinder moving in a shear flow close to a wall can be expressed as the sum of (2.1) and (2.2), i.e. as

$$
\left.\begin{array}{l}
\boldsymbol{F}^{*}=\mu^{*} \pi\left(-\sqrt{8 \frac{R^{*}}{\Delta^{*}}} V^{*}+4 R^{*} G^{*}\right) \boldsymbol{e}_{x} \\
\boldsymbol{T}^{*}=-\mu^{*} R^{* 2} \pi\left(\sqrt{8 \frac{R^{*}}{\Delta^{*}}} \Omega^{*}+6 G^{*}\right) \boldsymbol{e}_{z}
\end{array}\right\}
$$

(these are the two-dimensional analogues of the commonly used expressions derived for a sphere near a wall in a shear flow by Goldman et al. $(1967 a, b))$. In what follows, we expect that the dominant forces on the cylinder will be hydrodynamic; consequently, in (2.3), both terms in each bracket will be of the same order and $V^{*} / R^{*}$ and $\Omega^{*}$ can be expected to scale like $G^{*}\left(\Delta^{*} / R^{*}\right)^{1 / 2}$. In the absence of other forces and torques, the cylinder will slide over the wall and the relative speed between the bottom of the cylinder and the wall will be $V^{*}+R^{*} \Omega^{*}=-(1 / 2)\left(2 \Delta^{*} R^{*}\right)^{1 / 2} G^{*}$ (so that, in the frame of the wall, the base of the cylinder moves in the opposite direction to its centre).

\section{(b) The microscale problem: bond mechanics}

We now model the adhesive molecules and derive their contributions to the total force and torque exerted on the cylinder. With (2.3), the overall balance of forces and torques on the cylinder will be fully characterized in $§ 2 d$ below.

\section{(i) Model of the bonds}

For a given point $x_{0}^{*}$ on the wall, let $A_{\text {tot }} g^{*}\left(x_{0}^{*}, x^{*}, t^{*}\right) \delta x_{0}^{*} \delta x^{*}$ be the number of bonds, per unit length in the $z$-direction, that are attached between the segment bounded by $\left(x_{0}^{*}, 0\right)$ and $\left(x_{0}^{*}+\delta x_{0}^{*}, 0\right)$ on the wall and the segment bounded by $\left(x^{*}, h^{*}\left(x^{*}\right)\right)$ and $\left(x^{*}+\delta x^{*}, h^{*}\left(x^{*}+\delta x^{*}\right)\right)$ on the cylinder at time $t^{*}$, where $h^{*} \approx \Delta^{*}+(1 / 2) x^{* 2} / R^{*}$ (figure $\left.2 a\right)$. The total number of formed bonds per unit length in the transverse direction is $\iint_{-\infty}^{\infty} A_{\text {tot }} g^{*}\left(x_{0}^{*}, x^{*}, t^{*}\right) \mathrm{d} x^{*} \mathrm{~d} x_{0}^{*}$.

Following Dembo et al. (1988), the forward and reverse reaction rates for receptor-ligand binding are written as Boltzmann distributions, allowing highly stretched 'slip' bonds, for example, to be readily broken by thermal energy fluctuations. However, unlike Dembo et al., we assume that the bonds are allowed to subtend an angle $\alpha$ with the vertical direction (figure $2 a$ ). To account for this extra d.f., the forward rate is expressed as the probability density that a bond may form for a given value of $\alpha$ times the probability density that this geometrical configuration is realized in the unbound state. The probability densities of forming or breaking bonds between the wall at $\left(x_{0}^{*}, 0\right)$ and the 
(a)

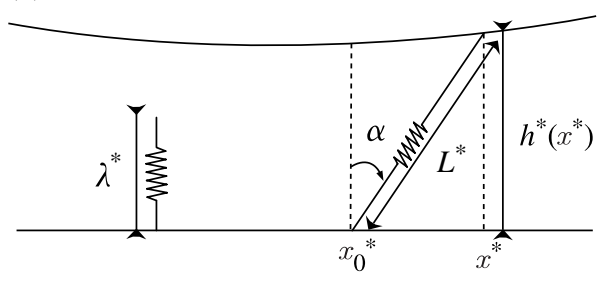

(b)

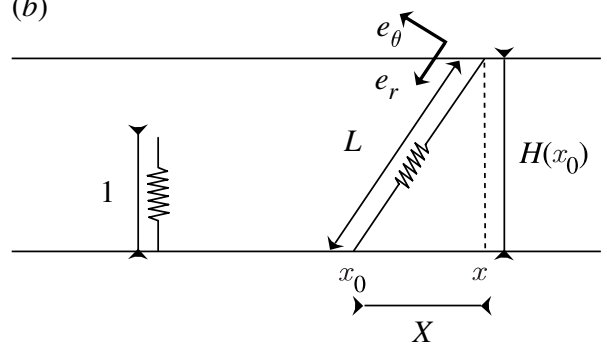

Figure 2. Model of the bonds between the wall and the cylinder. (a) Dimensional variables and (b) dimensionless quantities in the approximation where, at the scale of the bonds, the surface of the cylinder can be considered as flat.

cylinder at $\left(x^{*}, h^{*}\left(x^{*}\right)\right)$ (figure 2) are then assumed to be

$$
\begin{aligned}
& K_{\text {off }}\left(x_{0}^{*}, x^{*}\right)=K_{\text {off,eq }} \exp \left[\left(\kappa-\kappa_{\mathrm{ts}}\right)\left(L^{*}\left(x_{0}^{*}, x^{*}\right)-\lambda^{*}\right)^{2} / 2 k_{\mathrm{B}} \mathcal{T}\right], \\
& K_{\mathrm{on}}\left(x_{0}^{*}, x^{*}\right)=K_{\text {on,eq }} \exp \left[-\kappa_{\mathrm{ts}}\left(L^{*}\left(x_{0}^{*}, x^{*}\right)-\lambda^{*}\right)^{2} / 2 k_{\mathrm{B}} \mathcal{T}\right] \mathcal{P}\left(x^{*}, x_{0}^{*}\right),
\end{aligned}
$$

where $K_{\text {on,eq }}$ and $K_{\text {offeq }}$ are the forward and reverse reaction rates for an undisturbed bond; $L^{*}\left(x_{0}^{*}, x^{*}\right)=\left(h^{*}\left(x^{*}\right)^{2}+\left(x^{*}-x_{0}^{*}\right)^{2}\right)^{1 / 2}$ is the dimensional length of the bond; $\mathcal{P}\left(x_{0}^{*}, x^{*}\right)$ is the probability density that a free bond lies along the line between $x_{0}^{*}$ (on the wall) and $x^{*}$ (on the cylinder); $k_{\mathrm{B}}$ is Boltzmann's constant; $\mathcal{T}$ is the absolute temperature; $\kappa$ is the spring constant of one molecular bond; and $\kappa_{\mathrm{ts}}$ is the spring constant of the transition state (see Dembo et al. (1988)) used to distinguish catch $\left(\kappa<\kappa_{\mathrm{ts}}\right)$ from slip $\left(\kappa>\kappa_{\mathrm{ts}}\right)$ bonds.

We assume that the energy associated with tilting a bond from its vertical position is of the form $(1 / 2) \kappa_{\theta} \alpha^{2}$. Hence, in the spirit of Dembo et al.'s model, we assume a Boltzmann distribution for $\mathcal{P}$ of the form

$$
\mathcal{P}\left(x^{*}, x_{0}^{*}\right) \mathrm{d} x^{*}=\exp \left[-k_{\theta} \alpha^{2}\right] \frac{\mathrm{d} \alpha}{\mathcal{N}}, \quad \alpha=\arctan \left(\frac{x^{*}-x_{0}^{*}}{h^{*}\left(x^{*}\right)}\right), \quad k_{\theta}=\frac{\kappa_{\theta}}{2 k_{\mathrm{B}} \mathcal{T}} .
$$

Here $\mathcal{N}=\int_{-\pi / 2}^{\pi / 2} \exp \left[-k_{\theta} \xi^{2}\right] \mathrm{d} \xi=\left(\pi / k_{\theta}\right)^{1 / 2} \operatorname{erf}\left(\pi k_{\theta}^{1 / 2} / 2\right)$ is a normalization factor. The limit $k_{\theta} \rightarrow 0$ represents the limit in which the bonds are allowed to explore freely all possible angles under thermal fluctuations. For $k_{\theta} \rightarrow \infty$, all the bonds are restricted to the vertical, $\mathcal{P}\left(x^{*}, x_{0}^{*}\right) \rightarrow \delta\left(x^{*}-x_{0}^{*}\right)$, and no sliding can occur between the bound cylinder and the wall, as was assumed in the models of Dembo et al. (1988) and others.

(ii) Evolution equation for bond density

In the reference frame of the wall, the bottom of the cylinder moves at velocity

$$
\boldsymbol{V}_{\mathrm{b}}^{*}(x)=\left(V^{*}+\Omega^{*} R^{*}\right) \boldsymbol{e}_{x}+\mathcal{O}\left(\sqrt{\Delta^{*} / R^{*}}\right),
$$

which leads to tilting of consummated bonds from the vertical. In the limit of small binding affinity $\left(K_{\text {eq }}=A_{\text {tot }} K_{\text {on,eq }} / K_{\text {off,eq }} \ll 1\right)$ and assuming that receptors 
are abundant on the cylinder, the evolution equation for $g^{*}\left(x_{0}^{*}, x^{*}, t^{*}\right)$ is therefore

$$
\frac{\partial g^{*}}{\partial t^{*}}+\frac{\partial}{\partial x^{*}}\left[g^{*} \boldsymbol{V}_{\mathrm{b}}^{*} \cdot \boldsymbol{e}_{x}\right]=A_{\mathrm{tot}} K_{\mathrm{on}}-K_{\mathrm{off}} g^{*} .
$$

Equation (2.7) captures spontaneous bond formation (under thermal fluctuations that drive changes in the length and orientation of receptors) through $A_{\text {tot }} K_{\text {on }}$, bond breakage (mediated by force, for either catch or slip bonds) through $K_{\text {off }} g^{*}$ and bond tilting (through sliding of adjacent surfaces) through the advective term. As an upstream boundary condition on (2.7), we impose that the density of consummated bonds formed as $x^{*} \rightarrow \infty$ is zero (note that we expect $\boldsymbol{V}_{\mathrm{b}}^{*} \cdot \boldsymbol{e}_{x}<0$ ). In other words, there is no advection of bonds from far upstream. Neglecting competition for binding sites $\left(K_{\text {eq }} \ll 1\right)$ leaves $(2.7)$ linear in $g^{*}$, which simplifies the following analysis.

\section{(iii) Adhesive forces}

Let us consider one individual bond formed between location $\left(x_{0}^{*}, 0\right)$ on the substrate and $\left(x^{*}, h^{*}\right)$ on the cylinder. The instantaneous force it exerts on the cylinder has two components: an extensional force, which is related to bond stretching by Hooke's law $\boldsymbol{f}_{\mathrm{E}}^{*}=\kappa\left(L^{*}\left(x_{0}^{*}, x^{*}\right)-\lambda^{*}\right) \boldsymbol{e}_{r}$, and a torsional force, which is proportional to the angle formed by the bond with the vertical $\boldsymbol{f}_{\mathrm{T}}^{*}=\kappa_{\theta}\left(\left(1 / L^{*}\right) \arctan \left(\left(x^{*}-x_{0}^{*}\right) / h^{*}\right)\right) \boldsymbol{e}_{\theta}$. Here, $\boldsymbol{e}_{\boldsymbol{r}}$ and $\boldsymbol{e}_{\theta}$ are the radial and orthogonal unit vectors as shown in figure $2 b$. We define $\boldsymbol{F}_{\mathrm{E}}^{*}$ and $\boldsymbol{F}_{\mathrm{T}}^{*}$ to be the extensional and torsional forces (per unit length in the transverse direction) arising from all bonds, so that

$$
\boldsymbol{F}_{i}^{*}=A_{\text {tot }} \iint_{-\infty}^{\infty} g^{*}\left(x_{0}^{*}, x^{*}\right) \boldsymbol{f}_{i}^{*}\left(x_{0}^{*}, x^{*}\right) \mathrm{d} x^{*} \mathrm{~d} x_{0}^{*}, \quad i=\{E, T\} .
$$

Since the slope of the membrane in the contact region is of order $\mathcal{O}\left(x^{*} / R^{*}\right)$ and the bonds formed at a given location on the substrate interact with the membrane over a horizontal distance of $\mathcal{O}\left(\lambda^{*}\right)$, any variations in height that a single bond may experience scale like $\epsilon \lambda^{*}$, where $\epsilon=\lambda^{*} / R^{*} \ll 1$ (we do not consider highly elongated tethers). Thus, the surface of the cylinder can be considered to be locally flat. At each location on the substrate we therefore approximate $h^{*}$ and $L^{*}$ in $(2.4 a)$ and $(2.4 b)$ (figure $\left.2 b\right)$ as

$$
h^{*}\left(x^{*}\right) \approx h^{*}\left(x_{0}^{*}\right) \text { and } L^{*}\left(x_{0}^{*}, x^{*}\right) \approx\left(h^{*}\left(x_{0}^{*}\right)^{2}+\left(x^{*}-x_{0}^{*}\right)^{2}\right)^{1 / 2} .
$$

\section{(c) Non-dimensionalization}

Before assembling our expression for the total force on the cylinder, it is convenient to non-dimensionalize the problem.

We anticipate that, in general, $\delta \equiv \Delta^{*} / \lambda^{*}=\mathcal{O}(1)$. We therefore introduce the dimensionless variables

$$
\begin{aligned}
& \left.\begin{array}{l}
\left(x^{*}-x_{0}^{*}\right)=\lambda^{*} X, \quad x_{0}^{*}=\left(\lambda^{*} R^{*}\right)^{1 / 2} x_{0}, \\
h^{*}=\lambda^{*} H, \quad L^{*}=\lambda^{*} L, \quad t^{*}=t / G^{*},
\end{array}\right\} \\
& K_{\text {off }}=e_{\text {off }} K_{\text {off,eq }}, \quad K_{\text {on }}=e_{\text {on }} K_{\text {on,eq }} / \lambda^{*}, \quad V^{*}=V \lambda^{*} K_{\text {off,eq }} \text {, } \\
& g^{*}=g K_{\mathrm{eq}} / \lambda^{*}, \quad \Omega^{*}=\Omega \lambda^{*} K_{\mathrm{off}, \mathrm{eq}} / R^{*} \quad \text { and } \quad U=V+\Omega \text {. }
\end{aligned}
$$


In addition to the sliding velocity scale $\left(\lambda^{*} R^{*}\right)^{1 / 2} G^{*}$ identified in $\S 2 a,(2.7)$ reveals a second velocity scale (balancing bond tilting with bond breakage) $\lambda^{*} K_{\text {off.eq }}$, which we use as our reference scale in $(2.10 a)-(2.10 c)$. $U$ is then the dimensionless relative velocity between the wall and the base of the cylinder; in line with the argument presented in $\S 2 a$, we expect $U \leq 0$.

The shear rate and the physical properties of the bonds are conveniently described by the dimensionless parameters

$$
G=\frac{G^{*}}{\epsilon^{1 / 2} K_{\mathrm{off}, \mathrm{eq}}}, \quad \mathcal{C}=\frac{A_{\mathrm{tot}} K_{\mathrm{eq}} \kappa \lambda^{*}}{K_{\mathrm{off}, \mathrm{eq}} \mu^{*}}, \quad \gamma=\frac{\kappa \lambda^{* 2}}{k_{\mathrm{B}} \mathcal{T}} \quad \text { and } \quad \beta=\frac{\kappa_{\mathrm{ts}}}{\kappa},
$$

where $G$ is the dimensionless shear rate (the ratio of the two velocity scales); $\mathcal{C}$ is a visco-adhesive parameter (relating extensional bond forces (see (2.17) below) to hydrodynamic forces); $\gamma$ and $k_{\theta}$ (see (2.5)) compare bond stretching and torsion energy to thermal fluctuations, respectively; and $\beta$ is used to model the response of the bonds to extensional strain (following Dembo et al. 1988).

Non-dimensionalizing, the reaction rates $(2.4 a)$ and $(2.4 b)$, bond density evolution equation (2.7) and its boundary condition become

$$
\begin{aligned}
& e_{\mathrm{off}}= \exp \left[(1-\beta) \frac{\gamma}{2}\left(L\left(x_{0}, X\right)-1\right)^{2}\right], \\
& e_{\mathrm{on}}=\exp \left[-\beta \frac{\gamma}{2}\left(L\left(x_{0}, X\right)-1\right)^{2}-k_{\theta} \arctan ^{2}\left(\frac{X}{H}\right)\right] H / \mathcal{N} L^{2}, \\
& \epsilon^{1 / 2} G \frac{\partial g}{\partial t}=-U \frac{\partial g}{\partial X}+e_{\mathrm{on}}\left(x_{0}, X\right)-e_{\mathrm{off}}\left(x_{0}, X\right) g, \\
& g \rightarrow 0 \quad \text { as } X \rightarrow \infty \quad(\text { for } U<0) .
\end{aligned}
$$

In the limit $\epsilon^{1 / 2} G \ll 1$, we can neglect the first term in $(2.12 c)$ that accounts for non-equilibrium binding effects. The bonds are then assumed to form and break at a rate that is sufficiently rapid for them to remain in equilibrium, and we need only solve for $g\left(X ; x_{0}\right)$ satisfying

$$
U \frac{\partial g}{\partial X}=e_{\mathrm{on}}\left(x_{0}, X\right)-e_{\mathrm{off}}\left(x_{0}, X\right) g
$$

subject to $(2.12 d)$. Note that $x_{0}$ appears only parametrically in $g$ through

$$
L\left(x_{0}, X\right)=\left[H^{2}+X^{2}\right]^{1 / 2} \text { and } H=\delta+\frac{1}{2} x_{0}^{2} .
$$

Solutions of (2.13) are presented in $§ 3 a$ below (figure 3), from which extensional and torsional force densities per unit length (scaled on $A_{\text {tot }} K_{\mathrm{eq}} \kappa \lambda^{*}$ )

$$
\begin{aligned}
& \mathrm{F}_{\mathrm{E}}\left(x_{0}, U, \delta\right)=\int_{-\infty}^{\infty}-g\left(x_{0}, X\right)(1-1 / L)\left[X \boldsymbol{e}_{x}+H \boldsymbol{e}_{y}\right] \mathrm{d} X \\
& \mathrm{~F}_{\mathrm{T}}\left(x_{0}, U, \delta\right)=k_{\theta} / \gamma \int_{-\infty}^{\infty} g\left(x_{0}, X\right) H / L^{2} \arctan (X / H)\left[-\boldsymbol{e}_{x}+X / H \boldsymbol{e}_{y}\right] \mathrm{d} X,
\end{aligned}
$$

are computed (figure 4). 

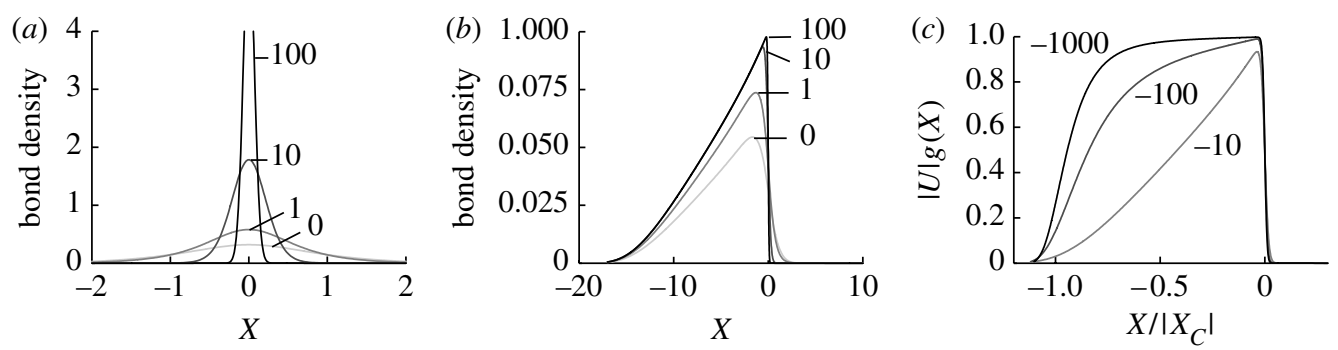

Figure 3. $(a)$ and $(b)$ show the effect of varying the spring constant $k_{\theta}$ (see labels in figures) on the bond density $g(X)$ for two sliding plates in the cases $U=0$ and -10 , respectively. (c) The effect of varying the sliding velocity $U$ (see labels in figure); here the rescaled bond density $|U| g(X)$ is plotted against $X /\left|X_{C}\right|$ for $k_{\theta}=10$, where $X_{C}=-\sqrt{2 \log |U| /(\gamma(1-\beta))}$. Other parameters are fixed $(H=1, \beta=0.98$ and $\gamma=1)$.
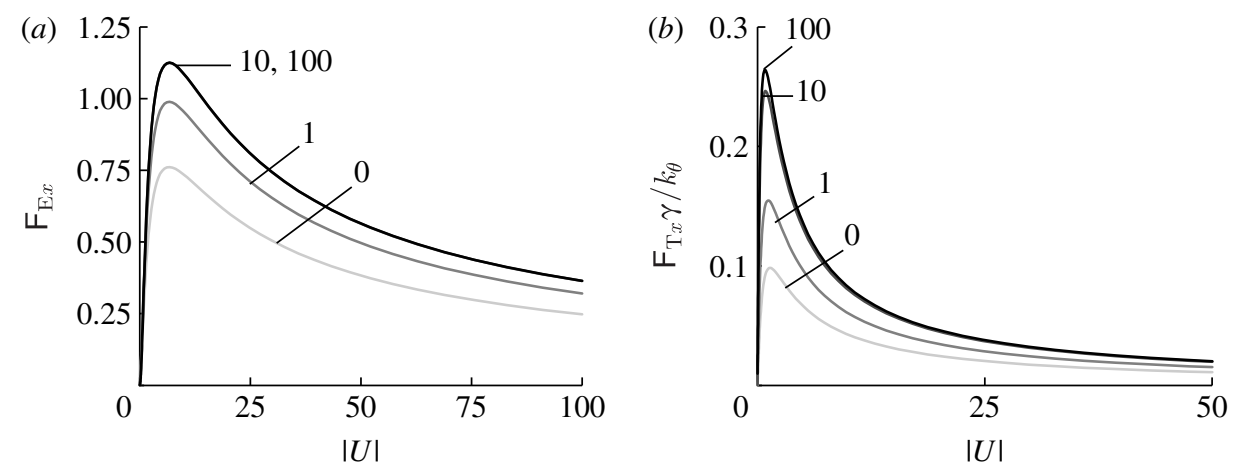

Figure 4. (a) Extensional force density $\mathrm{F}_{\mathrm{E} x}$ and $(b)$ torsional force density $\mathrm{F}_{\mathrm{T} x} \gamma / k_{\theta}$ between two sliding plates, as functions of the upper plate velocity $U$ (with $U<0$ ). Labels denote the spring constant $k_{\theta} ; H=1, \beta=0.9$ and $\gamma=1$.

\section{(d) Global force and torque balance}

The coupling between the macroscale and the microscale is obtained via the global force balance on the cylinder in the vertical and horizontal directions and the torque balance about the centre of mass of the cylinder. Assembling the contribution of adhesive forces from (2.8) and that of the fluid from (2.3), we obtain

$$
\begin{aligned}
& 0=\left(\boldsymbol{F}_{\mathrm{E}}^{*}+\boldsymbol{F}_{\mathrm{T}}^{*}\right) \cdot \boldsymbol{e}_{y}, \\
& 0=\left(\boldsymbol{F}_{\mathrm{E}}^{*}+\boldsymbol{F}_{\mathrm{T}}^{*}\right) \cdot \boldsymbol{e}_{x}-\mu^{*}\left(2 \sqrt{2} \pi V^{*}\left(R^{*} / \Delta^{*}\right)^{1 / 2}-4 \pi R^{*} G^{*}\right), \\
& 0=R^{*}\left(\boldsymbol{F}_{\mathrm{E}}^{*}+\boldsymbol{F}_{\mathrm{T}}^{*}\right) \cdot \boldsymbol{e}_{x}-\mu^{*} R^{* 2}\left(2 \sqrt{2} \pi \Omega^{*}\left(R^{*} / \Delta^{*}\right)^{1 / 2}+6 \pi G^{*}\right) .
\end{aligned}
$$

From $(2.10 a)-(2.10 c)$, the dimensionless forms of the net extensional and torsional forces are

$$
\boldsymbol{F}_{i}^{*}=A_{\mathrm{tot}} K_{\mathrm{eq}} \kappa \lambda^{*}\left(\lambda^{*} R^{*}\right)^{1 / 2} \boldsymbol{F}_{i}, \quad i=\{E, T\},
$$


where, using (2.15a) and (2.15b),

$$
\boldsymbol{F}_{\mathrm{E}}(U, \delta)=\int_{-\infty}^{\infty} \mathrm{F}_{\mathrm{E}}\left(x_{0}, U, \delta\right) \mathrm{d} x_{0}, \quad \boldsymbol{F}_{\mathrm{T}}(U, \delta)=\int_{-\infty}^{\infty} \mathrm{F}_{\mathrm{T}}\left(x_{0}, U, \delta\right) \mathrm{d} x_{0} .
$$

Rearranging $(2.16 a)-(2.16 c)$, non-dimensionalizing using $(2.10 a)-(2.10 c)$ and (2.11) and applying (2.17) yields

$$
\begin{gathered}
\left(\boldsymbol{F}_{\mathrm{E}}(U, \delta)+\boldsymbol{F}_{\mathrm{T}}(U, \delta)\right) \cdot \boldsymbol{e}_{y}=0, \\
\mathcal{C}\left(\boldsymbol{F}_{\mathrm{E}}(U, \delta)+\boldsymbol{F}_{\mathrm{T}}(U, \delta)\right) \cdot \boldsymbol{e}_{x}=\pi G+\sqrt{2} \pi \delta^{-1 / 2} U, \\
V=\frac{5 \sqrt{2 \delta}}{4} G+\frac{U}{2} .
\end{gathered}
$$

We treat the control parameters of the system that comprises $(2.13),(2.18)$ and $(2.19 a)-(2.19 c)$ as $G$ (dimensionless shear rate, see (2.11)), $k_{\theta}$ (resistance to tilting) and $\mathcal{C}$ (dimensionless adhesive force); and $V, U$ and $\delta$ (translation and sliding speeds and distance from wall) will be considered as output parameters. The dimensionless unstressed bond length $\epsilon \ll 1$ is fixed. To keep the analysis simple, we choose to keep the remaining intrinsic bond properties $\gamma$ and $\beta$ in $(2.12 a)$ and $(2.12 b)$ fixed and $\mathcal{O}(1)$.

We first solve for $g\left(X ; x_{0}\right)$ from $(2.13)$ as an integral involving $U, V$ and $\delta$ and then use (2.18) to determine $U, V$ and $\delta$ from (2.19a) and (2.19b). For given $k_{\theta}$, (2.19a) determines a relationship between $\delta$ and $U$, which can be written in the form $\delta=\delta(U)$, so that $(2.19 b)$ can be written

$$
G=(\mathcal{C} / \pi) \mathcal{F}(U)-\sqrt{2} U[\delta(U)]^{-1 / 2},
$$

where $\mathcal{F}(U)=F_{\mathrm{E} x}(U, \delta(U))+F_{\mathrm{T} x}(U, \delta(U))$ is determined by solving (2.13) for $g$ and substituting in (2.18). By solving (2.20) numerically, we obtain a relationship between the shear rate $G$ and the equilibrium sliding velocity $U$ for given $k_{\theta}$ and $\mathcal{C}$.

\section{Results}

\section{(a) Adhesion between two sliding parallel plates}

We start by considering the canonical microscale problem illustrated in figure $2 b$ in order to illustrate how the bond distribution and adhesion force densities depend on $k_{\theta}$ and $U$, considering only slip bonds, with $\beta<1$. Then we present numerical results for the full problem (figure 1).

\section{(i) Effects of $U$ and $k_{\theta}$ on bond distributions}

We first consider the formation of bonds between two infinite flat parallel plates, separated by a constant distance $H$, as the upper plate moves horizontally at speed $U \leq 0$. Here $L=L(X), e_{\mathrm{on}}=e_{\mathrm{on}}(X)$ and $e_{\mathrm{off}}=e_{\mathrm{off}}(X)$, so the solution to (2.13), under the rapid kinetics assumption, has the form $g=g(X)$.

For $U=0, g(X)=e_{\mathrm{on}}(X) / e_{\mathrm{off}}(X)$. The profile is symmetric and is proportional to a Dirac delta function as $k_{\theta} \rightarrow \infty$ (figure $3 a$ ). For non-zero sliding velocities $(U<0$, say), the reflection symmetry in $g$ is broken by advection (figure $3 b$ ). 
In this case, the analytical solution of (2.13) is

$$
g(X)=\frac{1}{|U|} \int_{X}^{\infty} e_{\text {on }}(t) \exp \left(-\frac{1}{|U|} \int_{X}^{t} e_{\text {off }}(s) \mathrm{d} s\right) \mathrm{d} t
$$

which has the limit (for $k_{\theta}^{-1 / 2} \ll|U|$ )

$$
g(X)= \begin{cases}0 & \text { if } X \geq 0 \\ \frac{1}{|U|} e_{\mathrm{on}}(0) \exp \left(-\frac{1}{|U|} \int_{X}^{0} e_{\mathrm{off}}(s) \mathrm{d} s\right) & \text { if } X<0 .\end{cases}
$$

This is graphically indistinguishable from the case $k_{\theta}=100$ in figure $3 b$. For $|U|$ large enough (typically $|U|>1$ ), the convergence to (3.2) is very fast, outside of a boundary layer of thickness $k_{\theta}^{-1 / 2}$ around $X=0$, within which (2.13) reduces at leading order to

$$
U g_{X}=e_{\text {on }}(0)\left(k_{\theta} / \pi\right)^{1 / 2} \exp \left[-k_{\theta} X^{2}\right]
$$

representing a balance between bond tilting and formation, with local solution $g=\left(e_{\mathrm{on}}(0) / 2|U|\right) \operatorname{erfc}\left(k_{\theta}^{1 / 2} X\right)$. For large $k_{\theta}$, the bond density therefore has a maximum of $\left|e_{\mathrm{on}}(0) / U\right|$ near $X \approx 0$ (figure $3 c$ ) and decays with a slope $e_{\mathrm{on}}(0) /|U|^{2}$ for $X \rightarrow 0^{-}$(see (3.2)). For sufficiently large $|U|$, this decay is small: bonds are swiftly advected downstream and have little time to be broken. However, their lifetime decreases exponentially with the square of their length (see $(2.12 a)$ ) so that the bond density decays super-exponentially fast to zero near $X_{C}=-\sqrt{2 \log |U| /(\gamma(1-\beta))}$, where the dominant balance in (2.13) becomes $U g_{X}=-\exp \left[(1-\beta)(\gamma / 2) X^{2}\right] g$, with local solution

$$
g=\frac{1}{|U|} \exp \left\{-\frac{1}{(1-\beta) \gamma\left|X_{C}\right|} \exp \left[-(1-\beta) \gamma\left|X_{C}\right|\left(X-X_{C}\right)\right]\right\},
$$

as $|U| \rightarrow \infty$. This behaviour is illustrated in figure $3 c$.

\section{(ii) Sliding friction}

In the same context, we now evaluate numerically the local force density (per unit area on the lower plate) exerted by the bonds formed between the two plates using $(2.15 a)$ and $(2.15 b)$.

The horizontal components $\mathrm{F}_{\mathrm{E} x} \equiv \mathrm{F}_{\mathrm{E}} \cdot \boldsymbol{e}_{x}$ and $\mathrm{F}_{\mathrm{T} x} \equiv \mathrm{F}_{\mathrm{T}} \cdot \boldsymbol{e}_{x}$ of the extensional and torsional force densities are given in figure 4 as functions of the relative velocity $U$. They vanish for $U=0$, by symmetry in the bonds' distribution (figure $3 a$ ), and initially increase with $U$ as advection stretches the bonds. As $|U| \rightarrow \infty$, however, bonds get stretched up to a length of order $X_{C} \propto \sqrt{\log |U|}$, but the maximum bond density decays like $|1 / U|$ (figure $3 c$ ) so that the resulting force density scales like $\log |U| /|U|$. Thus, the adhesive force reaches a maximum for $|U|=\mathcal{O}(1)$ and then decays to zero.

\section{(b) Cylinder in a shear flow}

We now consider the geometry specific to a cylinder (figure 1). Results are presented for $k_{\theta} \gg \gamma$, when the resistance of the bonds to tilting is large compared with that to stretching (i.e. near-vertical bonds). Figure 5 shows the 

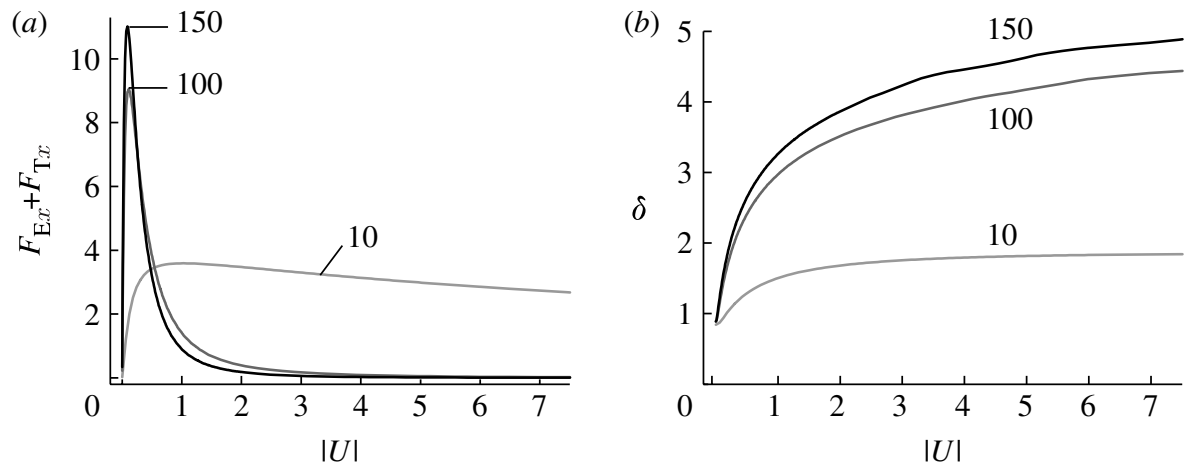

Figure 5. (a) Horizontal component of adhesive forces exerted on the cylinder $F_{\mathrm{E} x}+F_{\mathrm{T} x}$. (b) Effect of the sliding velocity $U$ on the vertical distance $\delta$ between the cylinder and the wall at equilibrium. Labels indicate different values of $k_{\theta} ; \beta=0.9$ and $\gamma=1$.

horizontal component of the adhesive force $F_{\mathrm{E} x}+F_{\mathrm{T} x}$ as a function of the sliding velocity $U$, as well as the height of the cylinder $\delta(U)$, for different values of $k_{\theta}$. Again, this force increases with $|U|$ for small $|U|$, as bonds are stretched, but decreases as $|U|$ increases further, due to bond breakage. Although this is a direct analogue of figure 4 , the force-velocity curve for the cylinder (figure $5 a$ ) exhibits a stronger nonlinear dependence on $k_{\theta}$ than for the plate, because $\delta$ varies as $|U|$ increases (figure $5 b$ ). Each bond that is advected away from its vertical position exerts a torsional force proportional to $k_{\theta}$ whose vertical component tends to push the cylinder away from the wall. For large $k_{\theta}$, this effect dominates the extensional forces in the stretched bonds (proportional to $\gamma$ ) that pull the cylinder closer to the wall.

Having determined the force on the cylinder, we now consider its kinematics. Figure $6 a$ shows the solution $(G, U)$ of $(2.20)$ for constant $k_{\theta}$ and $\mathcal{C}$, which shows the steady state of the system for any imposed shear rate $G$. As $G$ is increased from 0 to a critical value $G_{\mathcal{C}, 1}$ ( $\approx 115$ in this case), the absolute value of the sliding velocity $U$ remains very small but increases monotonically. In other words, the adhesive effects are strong and the cylinder rolls on the wall. However, when $G>G_{\mathcal{C}, 1}$ the system must jump to an equilibrium with a much larger value of $U$, comparable in magnitude with the sliding velocity reached in the absence of adhesive forces (a state we denote in figure $6 a$ as free sliding). If the shear rate is subsequently decreased, the cylinder resumes rolling as $G$ decreases through a lower critical value $G_{\mathcal{C}, 2}(\approx 78$ in this case).

Figure $6 b$ represents the height of the cylinder as $G$ varies. The cylinder is gradually pushed away from the wall as $G$ increases, with a discontinuous jump from $\delta \approx 1.5$ to 1.9 at the critical shear rate $G_{\mathcal{C}, 1}$. This jump is more pronounced when $k_{\theta}$ is large (from $\delta \approx 1.4$ to 5.5 for $k_{\theta}=100$ as shown in figure $7 b$ ). Increasing the bonds' resistance to tilting also increases the range of bistability between rolling and free sliding (figure $7 a$ ). The behaviour of the system depends crucially on the visco-adhesive parameter $\mathcal{C}$, defined in (2.11). Figure $8 a$ shows that hysteresis exists only for $\mathcal{C} \gtrsim 16$ when $k_{\theta}=10$. As $\mathcal{C}$ gets larger, the values of the critical shear rates $G_{\mathcal{C}, i}$ increase (quasi-linearly) as do the amplitudes of the jumps in velocity and height when $G$ crosses this threshold. 
(a)

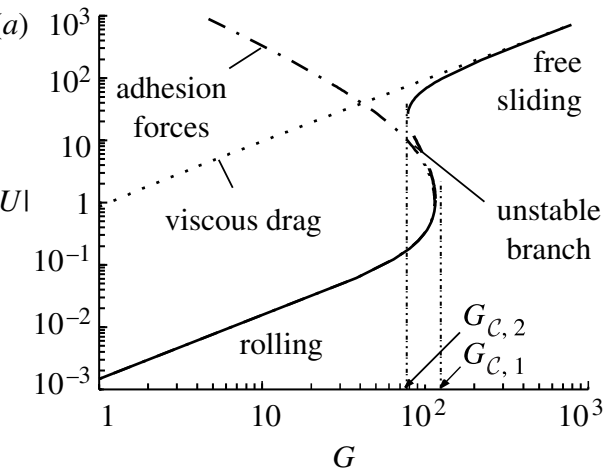

(b)

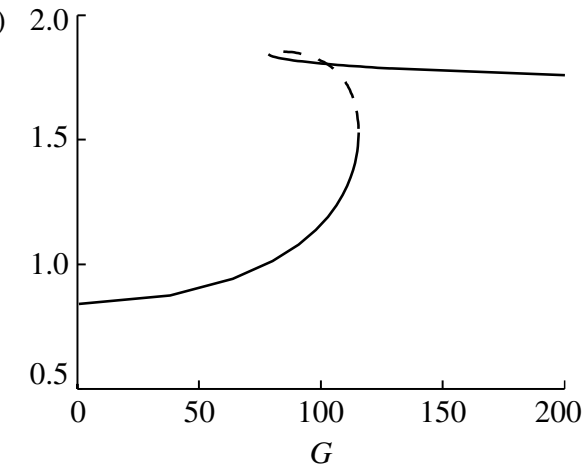

Figure 6. (a) Sliding velocity $|U|$ between the base of the cylinder and the wall as a function of the shear rate $G$ for a cylinder with $k_{\theta}=10$ and $\mathcal{C}=100$. The dot-dashed and dotted lines represent the contributions of the adhesive forces and hydrodynamic drag, respectively (first and second term of the r.h.s. of (2.20)). (b) Height $\delta$ of the cylinder as a function of the shear rate for the same value of $k_{\theta}$ and $\mathcal{C}$. The dashed line is the unstable branch. Other parameters are fixed: $\beta=0.9$ and $\gamma=1$.
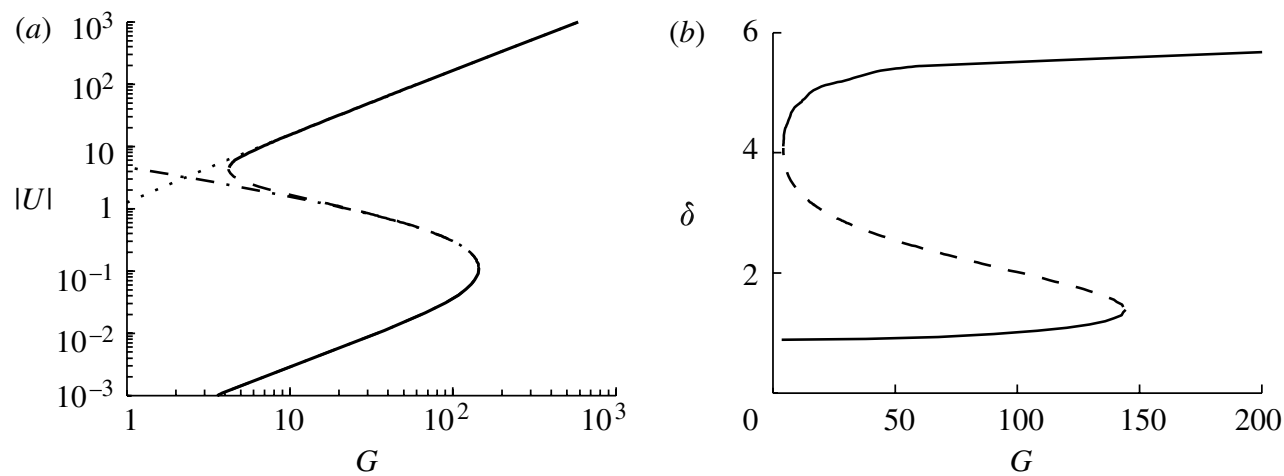

Figure 7. The descriptions are the same as given in figure legend 6 , except for $k_{\theta}=100$ and $\mathcal{C}=50$.

In summary, two different regimes can be identified: either the cylinder rolls on the wall (the bonds preventing sliding motion) or the cylinder is pushed away from the wall and is free from adhesive forces (with most of the bonds broken, yielding predominantly sliding motion). In $(G, \mathcal{C})$-parameter space, a hysteresis region exists where both regimes coexist and are (presumably) stable (figure $8 b$ ). The size of this bistability region increases with the bonds' resistance to tilting: as $k_{\theta} \rightarrow \infty$, for any given $\mathcal{C}, G_{\mathcal{C}, 1} \rightarrow \infty$ since the friction force that prevents a rolling cylinder from sliding tends to infinity, and $G_{\mathcal{C}, 2} \rightarrow 0$ since the sliding friction exerted on a free rolling cylinder tends to zero, so that it can only reattach if the shear rate vanishes. In contrast, the bistable region shrinks to a thin wedge in the region of large $\mathcal{C}$ and large $G$ as $k_{\theta} \rightarrow 0$ (the adhesion forces are then dominated by the extensional force $\boldsymbol{F}_{\mathrm{E}}$, which provides a sliding friction force independent of $k_{\theta}$ ).

The bistable region, when it exists, is bounded by two shear thresholds featuring abrupt transitions whereby the translation velocity of the centre of the 

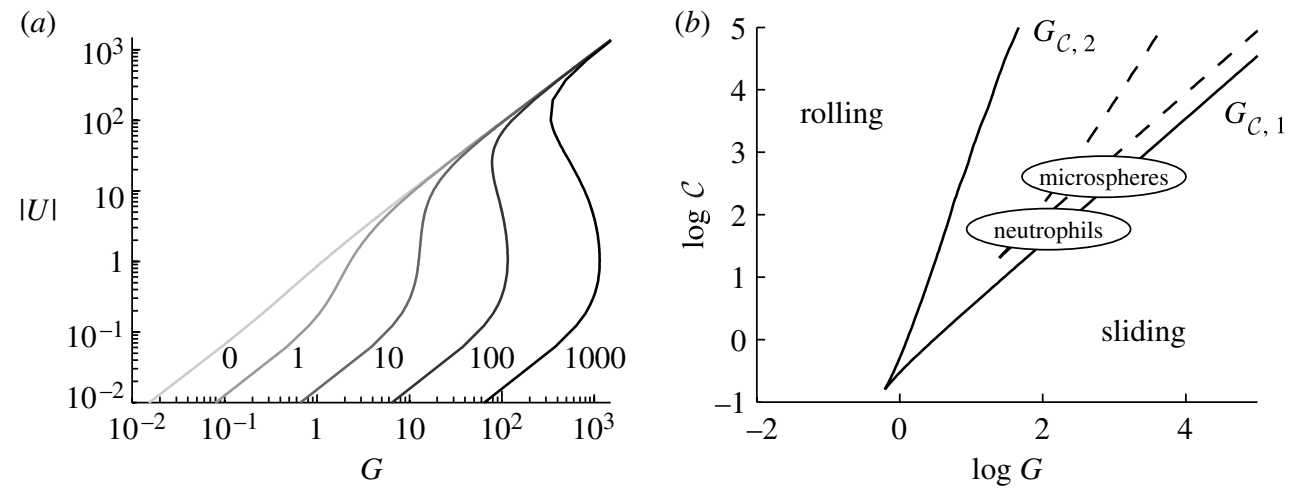

Figure 8. (a) Effects of the visco-adhesive parameter $\mathcal{C}$ (see labels on graph) on the relationship between sliding velocity and shear rate for $k_{\theta}=10$. (b) Behaviour of the cylinder in the $(G, \mathcal{C})$-plane. The lines separate two types of behaviours, with bistability in the wedged region. Solid, $k_{\theta}=100$; dashed, $k_{\theta}=10$. Ellipses illustrate the biological examples described in the discussion. Parameters used: $\beta=0.9$ and $\gamma=1$.

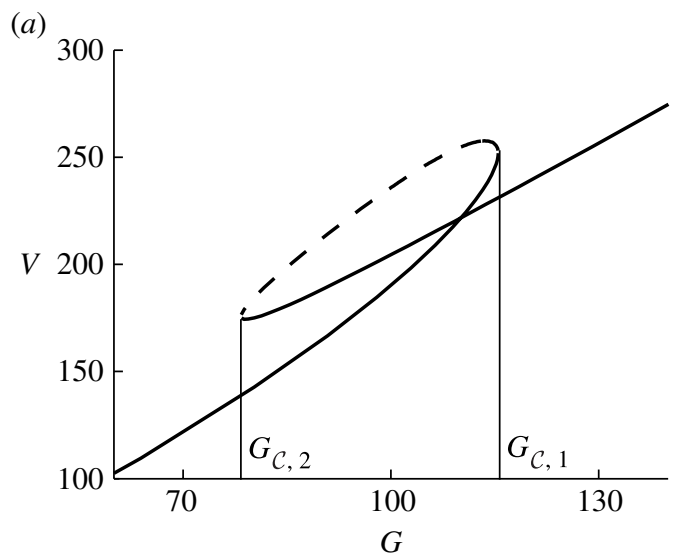

(b)

Figure 9. Translation velocity $V$ of the cylinder as a function of the dimensionless shear rate. (a) $k_{\theta}=10$ and $\mathcal{C}=100$ and $(b) k_{\theta}=100$ and $\mathcal{C}=50$. The dashed line is the unstable branch. The dotted line represents the case $k_{\theta} \rightarrow \infty$ with $\delta=0.88$. Other parameters are fixed: $\beta=0.9$ and $\gamma=1$.

cylinder $V$ undergoes a negative, or a positive, jump (figure 9) depending on parameters. Whether $V$ jumps up or down at $G_{\mathcal{C}, 1}$ depends on the competition in $(2.19 c)$ between $\delta$, which increases with $|U|$ and thus $G$ (figure $5 b$ ), and $U=-|U|$, which decreases.

Finally, it is worthwhile comparing these results to that from Dembo et al.'s (1988) model, in which bonds are assumed to be strictly vertical. As shown in appendix $\mathrm{A}$, for large but finite $k_{\theta}$ all the bonds form and break while almost vertical and the sliding speed $U \rightarrow 0$ : the cylinder rolls without sliding and its velocity depends (via $(2.19 c)$ ) on the equilibrium distance $\delta$ between the cylinder and the wall that arises in the absence of imposed shear. We find that $\delta$ is determined by both bond extension and (surprisingly) bond tilting (stiff bonds tilted through a small angle can generate a vertical force that balances that due 
to bond extension). The rolling speed with $\delta \approx 0.88$ (see figure $5 \mathrm{~b}$ with $U \rightarrow 0$ ) captures correctly the lower rolling solution branch for large finite $k_{\theta}$ (figure $9 b$ ). It is significant that weak bond tilting contributes at leading order to this limiting behaviour, implying that the limit $k_{\theta} \rightarrow \infty$ considered by Dembo et al. (1988) is singular.

\section{Discussion}

Continuum models for cell rolling along a wall in a shear flow often assume that the bonds between the cell and the wall remain vertical (e.g. Dembo et al. 1988). This delicate assumption (see appendix A) leads to the cell exhibiting a rolling motion in which there is no relative motion between the bound portion of the cell and the wall. In contrast, a rigid unbound cylinder (or a sphere), advected by a shear flow in proximity to a wall, exhibits significant sliding relative to the wall. To understand the role of sliding friction in cell adhesion, we have investigated a model in which we assumed that adhesive bonds exhibit a finite resistance to rotation. The hydrodynamic drag on the cell consequently results in bond tilting, allowing sliding of the cell over the wall. In order to avoid unnecessary complications, we conducted our study in two dimensions by investigating the motion of a rigid cylinder moving above a wall in a shear flow. In three dimensions (i.e. for a rigid sphere moving in a shear flow alongside a wall), we have found that the qualitative features of the dynamics are broadly similar (S. Reboux, G. Richardson \& O. E. Jensen 2007, unpublished results). The most notable difference comes from the scalings of the horizontal velocity arising from the hydrodynamic forcing $\left(\epsilon^{1 / 2} G^{*} R^{*}\right.$ and $G^{*} R^{*} /|\log \epsilon|$, respectively, for a cylinder and a sphere), leading to different time scales for bond advection from the leading edge to the trailing edge of the rolling cell. To assume equilibrium in binding kinetics, as we did for (2.7), this time scale has to be much larger than the time scale for bond breakage. In two dimensions, this occurs for $G^{*} \ll K_{\text {off,eq }}$, so that there is a well-defined range of shear rates for which the rapid kinetics assumption is valid. In three dimensions, the condition becomes $G^{*} \ll \epsilon^{1 / 2}|\log \epsilon| K_{\text {off,eq }}$, which is more restrictive as $\epsilon \ll 1$. Even so, we have found that accounting for non-equilibrium binding kinetics in three dimensions preserves, at least qualitatively, the results shown in the present study.

Despite its relative simplicity, our model is capable of describing a number of different behaviours which have not been captured previously (even in models that accommodate bond tilting) and which arise as a result of the coupling between viscous forces at the macroscale level and friction at molecular scales. We started by exploring the effects of the frictional forces arising from the molecular binding between two parallel sliding surfaces $(\$ 3 a)$. We found that at low sliding velocities, the bonds are arranged almost symmetrically (figure $3 a$ ) so that the average horizontal frictional force is almost zero. As the sliding velocity increases the bonds tilt in the direction of the motion, giving rise to a much increased resistance to sideways motion (figures $3 b$ and 4 ) that initially increases linearly with $|U|$. However, further increases in the sliding velocity lead to significant bond extension and breakage and a corresponding decrease in the (frictional) resistance to motion (figure 4), with the frictional force falling like $\log |U| /|U|$. Such behaviour may 
be relevant to biomimetic adhesives involving independent flexible pillars that randomly bind to and break from a nearby moving surface (Varenberg \& Gorb 2007).

When such behaviour is included in our model for the motion of a cylinder $(\S 3 b)$, one of the most interesting features is the nonlinear relation between the (dimensionless) sliding velocity $|U|$ and the (dimensionless) shear rate $G$ which can give rise to hysteresis (depending on the bonds' resistance to tilting $k_{\theta}$ and the visco-adhesive parameter $\mathcal{C}$ ). This is characterized by a rolling solution branch (with $|U| \ll 1$ ) at small shear rate and a sliding solution branch (where the motion is largely unaffected by the bonds between cell and wall) at large shear rate (figure 6). These two branches are joined by a third solution branch via two folds, so that, as the shear is increased from a low value through a critical value $G_{c, 1}$, an abrupt transition occurs between the rolling solution and the free sliding solution. Likewise, as the shear is decreased from a high value of $G$ through a second critical value $G_{c, 2}$ (where $\left.G_{c, 2}<G_{c, 1}\right)$, an abrupt transition occurs between sliding and rolling. This hysteresis occurs as a result of two competing effects, namely the hydrodynamic drag experienced by the cell in shear flow (which dominates at high shear rates) and the sliding friction due to intermolecular bonds, which dominates at low shear rates, resulting in a characteristic ' $\mathrm{S}$ '-shaped curve (see figure $8 a$ for $\mathcal{C}>16$ ). For relatively weak bonds (i.e. a low visco-adhesive parameter $\mathcal{C}$ ), the viscous drag always dominates and non-hysteretic behaviour results (see figure $8 a$ for $\mathcal{C}<16$ ). Physically, we can explain the abrupt hysteretic transition (where it occurs) between rolling and sliding solutions by noting that, as the shear is increased, the bonds become unable to prevent some degree of sliding between the base of the cylinder and the wall and start to yield. In turn this decreases the total adhesive force, further increasing the sliding velocity and eventually leading to a state where the cylinder is free from nearly all adhesive bonds.

The domain of bistability that we have characterized lies within the range of physiological parameters for several biological systems involving cell-wall adhesion. Owing to both the variety of such systems and the difficulty of measuring some of the parameters experimentally, the dimensionless quantities introduced in our model are likely to cover several orders of magnitude in different physiological conditions. Broadly, we estimate that both the dimensionless shear rate $G$ and the visco-adhesive parameter $\mathcal{C}$ vary between $10^{1}$ and $10^{4}$ (table 1 ). These ranges are obtained by considering a cell of radius $R^{*}=4 \mu \mathrm{m}$ (e.g. a white blood cell) interacting with a flat surface via P-selectin/ PSGL-1 bonds. We also account for the fact that the mechanical properties of the bonds can be modified by the presence of extensible microvilli between the PSGL-1 receptors and the cell (Shao et al. 1998). Let us consider two examples

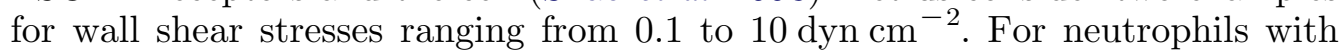
microvilli rolling over a surface coated with P-selectin at $10^{2}$ sites $\mu \mathrm{m}^{-2}$, we have $\mathcal{C} \approx 350$ and $G$ within $10^{1}-10^{3}$. For rigid microspheres rolling over P-selectin at 10 sites $\mu \mathrm{m}^{-2}$, the spring constant $\kappa^{*}$ is much higher and the bonds are much shorter (no microvilli). We then have $\mathcal{C} \approx 80$ and $G$ within $7 \times 10^{1}-7 \times 10^{3}$. Note that $\mathcal{C}$ is proportional to $\mathrm{P}$-selectin density and binding affinity. In figure 8 we report these values on the $(G, \mathcal{C})$-diagram for our model of a cylinder in a shear 
Table 1. Ranges of physiological parameters.

\begin{tabular}{|c|c|c|c|}
\hline symbol & definition & range & references \\
\hline$R^{*}$ & cell radius & $4.0 \mu \mathrm{m}$ & Schmidt-Shönbein et al. (1975) \\
\hline$\lambda^{*}$ & bond length & $10-300 \mathrm{~nm}$ & Springer (1990) and Shao et al. (1998) \\
\hline$G^{*}$ & shear rate & $40-2000 \mathrm{~s}^{-1}$ & \\
\hline$A_{\text {tot }}$ & receptor density & $10-10^{2} \mu \mathrm{m}^{-2}$ & Lawrence \& Springer (1991) \\
\hline$K_{\text {off.eq }}^{*}$ & reverse rate & $1-10 \mathrm{~s}^{-1}$ & Bell (1978) and Rinko et al. (2004) \\
\hline$K_{\mathrm{on}, \mathrm{eq}}^{*}$ & forward rate & $1-100 \mathrm{~s}^{-1}$ & $\begin{array}{l}\text { Lawrence \& Springer (1991) and Rinko } \\
\text { et al. (2004) }\end{array}$ \\
\hline$\kappa^{*}$ & spring constant & $0.01-5$ dyn $\mathrm{cm}^{-1}$ & Fritz et al. (1998) and Shao et al. (1998) \\
\hline$k_{\theta}$ & resistance to bending & $0-10^{3}$ & \\
\hline$G$ & shear rate & $1-10^{4}$ & \\
\hline $\mathcal{C}$ & visco-adhesive parameter & $1-10^{4}$ & \\
\hline
\end{tabular}

flow. We have conducted a detailed analysis for the three-dimensional case of a sphere in a shear flow and, in physiological parameter regimes, obtained the same qualitative features as in the two-dimensional case (results will be presented elsewhere).

There are obviously a number of limitations to the approach we have adopted. These include the effects of cell deformability (significant in many cell-adhesion applications (Jadhav et al. 2005; Khismatullin \& Truskey 2005; Jin et al. 2007)), which introduces a lift term in the force balance and thereby modifies the extent of the adhesion region. In addition our approach excludes spatial inhomogeneities, either of material properties or arising spontaneously (Brener et al. 2005). We have considered one particular class of model for intermolecular bonds; the effects of catch behaviour $(\beta>1)$, non-equilibrium effects, stochasticity and discrete bond numbers certainly deserve further attention, and in some applications such effects may mask the bistable behaviour considered here. In particular, our steady-state analysis (along with the notion of bistability itself) becomes irrelevant when considering systems with a relatively small number of bonds where high levels of stochasticity induce fluctuations between different macrostates and no steady state is ever observed (Hammer \& Apte 1992), and so experiments are most likely to exhibit the predicted bistability when multiple bonds simultaneously mediate adhesion. Finally, our model accounts neither for receptor and ligand migration on the wall and cell surfaces (Lawrence \& Springer 1991) nor for forces large enough to tear ligands or receptors from their anchoring (Shao \& Hochmuth 1999).

In conclusion, for a rigid cylinder in a shear flow adhering to the wall via tiltable tethers that permit sliding friction, we have demonstrated the existence, under certain conditions, of two critical shear rates, $G_{\mathcal{C}, 1}>G_{\mathcal{C}, 2}$, between which the system has two equilibrium states: the cell either rolls on the surface or moves freely at its hydrodynamic speed. If the dimensionless shear rate $G$ is increased above $G_{\mathcal{C}, 1}$, most of the bonds break. If $G$ is decreased below $G_{\mathcal{C}, 2}$, they reform and the cell rolls. These results are likely to be generic and the bistable region falls in the range of several biological applications. Nonlinearity in sliding friction can lead to non-trivial and non-intuitive effects which deserve deeper investigation. 


\section{Appendix A. Comparison with Dembo's et al.'s model}

Here we investigate the limit $U \ll k_{\theta}^{-1 / 2} \ll 1$, which models bonds that form and break while almost vertical, but when the high resistance of bonds to tilting provides a sideways force sufficient to restrict sliding significantly. We pose an expansion $g \approx g_{0}+U g_{1}$ and substitute in (2.13) to obtain $g_{0}=e_{\mathrm{on}}(X) / e_{\text {off }}(X)$, $g_{1}=g_{0 X} / e_{\text {off }}(X)$, which in turn can be substituted in the expressions for the adhesive force densities $(2.15 a)$ and $(2.15 b)$. These can likewise be written in the form.

$$
\mathrm{F}_{\mathrm{E}}\left(k_{\theta}\right) \approx \mathrm{F}_{\mathrm{E} 0}\left(k_{\theta}\right)+U \mathrm{~F}_{\mathrm{E} 1}\left(k_{\theta}\right) \text { and } \mathrm{F}_{\mathrm{T}}\left(k_{\theta}\right) \approx \mathrm{F}_{\mathrm{T} 0}\left(k_{\theta}\right)+U \mathrm{~F}_{\mathrm{T} 1}\left(k_{\theta}\right) .
$$

For $k_{\theta} \gg 1$, writing $u=k_{\theta}^{1 / 2} \arctan (X / H)$, we can express $g_{0}(X)$ as proportional to a Gaussian $\mathrm{e}^{-u^{2}}$, which in the limit $k_{\theta} \rightarrow \infty$ behaves like a $\delta$-function, so that $\mathrm{F}_{\mathrm{E} 0}$ converges to the value of the integrand in $(2.15 a)$ for $X=0$.

$$
\mathrm{F}_{\mathrm{E} 0}=\left(1-H\left(x_{0}\right)\right) \exp \left(-(\gamma / 2)\left(H\left(x_{0}\right)-1\right)^{2}\right) \boldsymbol{e}_{y} .
$$

The integral for $\mathbf{F}_{\mathrm{T} 0}$ from $(2.15 b)$ reduces in the limit to the form $\int u^{2} \mathrm{e}^{-u^{2}} \mathrm{~d} u$, yielding

$$
\mathbf{F}_{\mathrm{T} 0}=\frac{\exp \left(-(\gamma / 2)\left(H\left(x_{0}\right)-1\right)^{2}\right)}{2 \gamma H\left(x_{0}\right)} \boldsymbol{e}_{y} .
$$

From symmetry, $\mathrm{F}_{\mathrm{E} 0} \cdot \boldsymbol{e}_{x}=\mathrm{F}_{\mathrm{T} 0} \cdot \boldsymbol{e}_{x}=0$. Physically, bonds form with a density $g(X)=\mathcal{O}\left(k_{\theta}^{1 / 2}\right)$ over a length scale $X=\mathcal{O}\left(k_{\theta}^{-1 / 2}\right)$. An $\mathcal{O}(1)$ number of bonds are tilted by an angle $\mathcal{O}\left(k_{\theta}^{-1 / 2}\right)$, exerting a (mostly horizontal) force of order $k_{\theta}^{1 / 2}$ that has an $\mathcal{O}(1)$ vertical contribution.

Since the value of $U$ is determined by the horizontal force balance $(2.19 b)$, it depends on $F_{\mathrm{E} 1}$ and $\mathrm{F}_{\mathrm{T} 1}$. An analysis similar to that conducted above (noting that $\left.g_{1}=\mathcal{O}\left(k_{\theta}^{1 / 2} g_{0}\right)\right)$ leads to.

$$
\mathrm{F}_{\mathrm{E} 1} \sim \mathcal{O}(1) \boldsymbol{e}_{x}+\mathcal{O}\left(k_{\theta}^{1 / 2}\right) \boldsymbol{e}_{y} \quad \text { and } \quad \mathrm{F}_{\mathrm{T} 1} \sim \mathcal{O}\left(k_{\theta}\right) \boldsymbol{e}_{x}+\mathcal{O}\left(k_{\theta}^{1 / 2}\right) \boldsymbol{e}_{y} .
$$

The horizontal force balance on the cylinder is dominated by shear $\pi G$ and bonds resisting tilting $\mathrm{F}_{\mathrm{T} 1}$, implying that $U \sim \mathcal{O}\left(G / k_{\theta}\right)$ (which, for $G=O(1)$, is consistent with the initial assumption $U \ll k_{\theta}^{-1 / 2}$ ). Substituting (A 4) into (A 1) and (2.19b) confirms that $\mathrm{F}_{\mathrm{E}} \cdot \boldsymbol{e}_{y} \sim \mathrm{F}_{\mathrm{E} 0} \cdot \boldsymbol{e}_{y}+\mathcal{O}\left(k_{\theta}^{-1 / 2}\right)$ and $\mathrm{F}_{\mathrm{T}} \cdot \boldsymbol{e}_{y} \sim \mathrm{F}_{\mathrm{T} 0} \cdot \boldsymbol{e}_{y}+\mathcal{O}\left(k_{\theta}^{-1 / 2}\right)$.

Thus for $k_{\theta} \rightarrow \infty$, in the presence of $O(1)$ shear, the cylinder rolls without sliding with $U \rightarrow 0$. Its velocity depends on the distance $\delta$ between the cylinder and the wall $(2.19 c)$. This is determined by the vertical force balance $(2.19 a)$, involving contributions from both bond compression and tilting, which are, respectively,

$$
\left.\begin{array}{l}
\boldsymbol{F}_{\mathrm{E}} \cdot \boldsymbol{e}_{\boldsymbol{y}}=\int_{-\infty}^{\infty}\left(1-H\left(x_{0}\right)\right) \exp \left(-(\gamma / 2)\left(H\left(x_{0}\right)-1\right)^{2}\right) \mathrm{d} x_{0}, \\
\boldsymbol{F}_{\mathrm{T}} \cdot \boldsymbol{e}_{\boldsymbol{y}}=\int_{-\infty}^{\infty} \frac{\exp \left(-(\gamma / 2)\left(H\left(x_{0}\right)-1\right)^{2}\right)}{2 H\left(x_{0}\right)} \mathrm{d} x_{0} .
\end{array}\right\}
$$

In a naive implementation of Dembo et al.'s model, it would be natural to consider only the contribution from $\boldsymbol{F}_{\mathrm{E}} \cdot \boldsymbol{e}_{y}$ above, yielding the equilibrium value $\delta \approx 0.24$. However, the contribution from bond tilting $\boldsymbol{F}_{\mathrm{T}} \cdot \boldsymbol{e}_{y}$ repels the cylinder further from the wall at equilibrium, giving $\delta \approx 0.88$, consistent with figure $5 b$. 


\section{References}

Alon, R., Hammer, D. A. \& Springer, T. A. 1995 Lifetime of the P-selectin-carbohydrate bond and its response to tensile force in hydrodynamic flow. Nature 374, 539-542. (doi:10.1038/374539a0)

Alon, R., Chen, S. Q., Puri, K. D., Finger, E. B. \& Springer, T. A. 1997 The kinetics of L-selectin tethers and the mechanics of selectin-mediated rolling. J. Cell Biol. 138, 1169-1180. (doi:10. 1083/jcb.138.5.1169)

Andersson, M., Fallman, E., Uhlin, B. E. \& Axner, O. 2006 A sticky chain model of the elongation and unfolding of Escherichia coli $\mathrm{P}$ pili under stress. Biophys. J. 90, 1521-1534. (doi:10.1529/ biophysj.105.074674)

Bell, G. I. 1978 Models for the specific adhesion of cells to cells. Science 200, 618-627. (doi:10.1126/ science.347575)

Brener, E. A., Malinin, S. V. \& Marchenko, V. I. 2005 Fracture and friction: stick-slip motion. Eur. Phys. J. E 17, 101-113. (doi:10.1140/epje/i2004-10112-3)

Caputo, K. E., Lee, D., King, M. R. \& Hammer, D. A. 2007 Adhesive dynamics simulations of the shear threshold effect for leukocytes. Biophys. J. 92, 787-797. (doi:10.1529/biophysj.106. 082321)

Chang, K. C. \& Hammer, D. A. 1999 The forward rate of binding of surface-tethered reactants: effect of relative motion between two surfaces. Biophys. J. 76, 1280-1292.

Dembo, M., Torney, D. C., Saxman, K. \& Hammer, D. 1988 The reaction-limited kinetics of membrane-to-surface adhesion and detachment. Proc. R. Soc. B 234, 55-83. (doi:10.1098/rspb. 1988.0038)

Dong, C. \& Lei, X. X. 2000 Biomechanics of cell rolling: shear flow, cell-surface adhesion, and cell deformability. J. Biomech. 33, 35-43. (doi:10.1016/S0021-9290(99)00174-8)

Drummond, C., Israelachvili, J. \& Richetti, P. 2003 Friction between two weakly adhering boundary lubricated surfaces in water. Phys. Rev. E 67, 66110. (doi:10.1103/PhysRevE.67. 066110)

Evans, E. \& Ritchie, K. 1997 Dynamic strength of molecular adhesion bonds. Biophys. J. 72, $1541-1555$.

Filippov, A. E. \& Popov, V. 2007 Flexible tissue with fibres interacting with an adhesive surface. J. Phys. Condens. Matter 19, 096012. (doi:10.1088/0953-8984/19/9/096012)

Filippov, A. E., Klafter, J. \& Urbakh, M. 2004 Friction through dynamical formation and rupture of molecular bonds. Phys. Rev. Lett. 92, 135503. (doi:10.1103/PhysRevLett.92.135503)

Finger, E. B., Puri, K. D., Alon, R., Lawrence, M. B., von Andrian, U. H. \& Springer, T. A. 1996 Adhesion through L-selectin requires a threshold hydrodynamic shear. Nature 379, 266-269. (doi:10.1038/379266a0)

Fritz, J., Katopodis, A. G., Kolbinger, F. \& Anselmetti, D. 1998 Force-mediated kinetics of single P-selectin ligand complexes observed by atomic force microscopy. Proc. Natl Acad. Sci. USA 95, 12 283-12 288. (doi:10.1073/pnas.95.21.12283)

Goldman, A. J., Cox, R. G. \& Brenner, H. 1967 a Slow viscous motion of a sphere parallel to a plane wall-I. Motion through a quiescent fluid. Chem. Eng. Sci. 22, 637-651. (doi:10.1016/0009$2509(67) 80047-2)$

Goldman, A. J., Cox, R. G. \& Brenner, H. $1967 b$ Slow viscous motion of a sphere parallel to a plane wall-II. Couette flow. Chem. Eng. Sci. 22, 653-660. (doi:10.1016/0009-2509(67)80048-4)

Gravish, N., Wilkinson, M. \& Autumn, K. In press. Frictional and elastic energy in gecko adhesive detachment. J. R. Soc. Interface. (doi:10.1098/rsif.2007.1077)

Hammer, D. A. 2005 Leukocyte adhesion: what's the catch? Curr. Biol. 15, R96-R99. (doi:10. 1016/j.cub.2005.01.028)

Hammer, D. A. \& Apte, S. M. 1992 Simulation of cell rolling and adhesion on surfaces in shearflow-general results and analysis of selectin-mediated neutrophil adhesion. Biophys. J. 63, $35-57$.

Hodges, S. R. \& Jensen, O. E. 2002 Spreading and peeling dynamics in a model of cell adhesion. J. Fluid Mech. 460, 381-409. (doi:10.1017/S0022112002008340) 
Isberg, R. R. \& Barnes, P. 2002 Dancing with the host: flow-dependent bacterial adhesion. Cell 110, 1-4. (doi:10.1016/S0092-8674(02)00821-8)

Jadhav, S., Eggleton, C. D. \& Konstantopoulos, K. 2005 A 3-D computational model predicts that cell deformation affects selectin-mediated leukocyte rolling. Biophys. J. 88, 96-104. (doi:10. 1529/biophysj.104.051029)

Jeffrey, D. J. \& Onishi, Y. 1981 The slow motion of a cylinder next to a plane wall. Q. J. Mech. Appl. Math. 34, 129-137. (doi:10.1093/qjmam/34.2.129)

Jin, Q., Verdier, C., Singh, P., Aubry, N., Chotard-Ghodsnia, R. \& Duperray, A. 2007 Migration and deformation of leukocytes in pressure driven flows. Mech. Res. Commun. 34, 411-422. (doi:10.1016/j.mechrescom.2007.07.002)

Khismatullin, D. B. \& Truskey, G. A. 2005 Three-dimensional numerical simulation of receptormediated leukocyte adhesion to surfaces: effects of cell deformability and viscoelasticity. Phys. Fluids 17, 31505. (doi:10.1063/1.1862635)

Krasik, E. F., Yee, K. L. \& Hammer, D. A. 2006 Adhesive dynamics simulation of neutrophil arrest with deterministic activation. Biophys. J. 91, 1145-1155. (doi:10.1529/biophysj.105.070706)

Lawrence, M. B. \& Springer, T. A. 1991 Leukocytes roll on a selectin at physiologic flow rates: distinction from and prerequisite for adhesion through integrins. Cell 65, 859-873. (doi:10.1016/ 0092-8674(91)90393-D)

Liotta, L. A. 2001 Cancer - an attractive force in metastasis. Nature 410, 24-25. (doi:10.1038/ $35065180)$

Marshall, B. T., Long, M., Piper, J. W., Yago, T., McEver, R. P. \& Zhu, C. 2003 Direct observation of catch bonds involving cell-adhesion molecules. Nature 423, 190-193. (doi:10. 1038/nature01605)

McEver, R. P. 2001 Adhesive interactions of leukocytes, platelets, and the vessel wall during hemostasis and inflammation. Thromb. Haemostasis 86, 746-756.

Moore, N. W. \& Kuhl, T. L. 2006 The role of flexible tethers in multiple ligand-receptor bond formation between curved surfaces. Biophys. J. 91, 1675-1687. (doi:10.1529/biophysj.105. 079871)

N'dri, N. A., Shyy, W. \& Tran-Son-Tay, R. 2003 Computational modeling of cell adhesion and movement using a continuum-kinetics approach. Biophys. J. 85, 2273-2286.

Ramachandran, V., Williams, M., Yago, T., Schmidtke, D. W. \& McEver, R. P. 2004 Dynamic alterations of membrane tethers stabilize leukocyte rolling on P-selectin. Proc. Natl Acad. Sci. USA 101, 13 519-13 524. (doi:10.1073/pnas.0403608101)

Rinko, L. J., Lawrence, M. B. \& Guilford, W. H. 2004 The molecular mechanics of P- and L-selectin lectin domains binding to PSGL-1. Biophys. J. 86, 544-554.

Rychak, J. J., Lindner, J. R., Ley, K. \& Klibanov, A. L. 2006 Deformable gas-filled microbubbles targeted to P-selectin. J. Control. Release 114, 288-299. (doi:10.1016/j.jconrel.2006.06.008)

Schmidtke, D. W. \& Diamond, S. L. 2000 Direct observation of membrane tethers formed during neutrophil attachment to platelets or P-selectin under physiological flow. J. Cell Biol. 149, 719-729. (doi:10.1083/jcb.149.3.719)

Schmidt-Shönbein, G. W., Fung, Y. C. \& Zweifach, W. 1975 Vascular endothelium-leukocyte interaction, sticking shear force in venules. Circ. Res. 36, 173-184.

Schubert, G. 1967 Viscous flow near a cusped corner. J. Fluid Mech. 27, 647-656. (doi:10.1017/ S0022112067002526)

Shao, J. Y. \& Hochmuth, R. M. 1999 Mechanical anchoring strength of L-selectin, $\beta_{2}$ integrins, and CD45 to neutrophil cytoskeleton and membrane. Biophys. J. 77, 587-596.

Shao, J. Y., Ting-Beall, H. P. \& Hochmuth, R. M. 1998 Static and dynamic lengths of neutrophil microvilli. Proc. Natl Acad. Sci. USA 95, 6797-6802. (doi:10.1073/pnas.95.12.6797)

Springer, T. A. 1990 Adhesion receptors of the immune-system. Nature 346, 425-434. (doi:10.1038/ $346425 \mathrm{a} 0)$

Sun, C. H., Migliorini, C. \& Munn, L. L. 2003 Red blood cells initiate leukocyte rolling in postcapillary expansions: a lattice Boltzmann analysis. Biophys. J. 85, 208-222. 
Tees, D. F. J., Waugh, R. E. \& Hammer, D. A. 2001 A microcantilever device to assess the effect of force on the lifetime of selectin-carbohydrate bonds. Biophys. J. 80, 668-682.

Urbakh, M., Klafter, J., Gourdon, D. \& Israelachvili, J. 2004 The nonlinear nature of friction. Nature 430, 525-528. (doi:10.1038/nature02750)

Varenberg, M. \& Gorb, S. 2007 Shearing of fibrillar adhesive microstructure: friction and shearrelated changes in pull-off force. J. R. Soc. Interface 4, 721-725. (doi:10.1098/rsif.2007.0222)

Yago, T., Zarnitsyna, V. I. Z., Klopocki, A. G., McEver, R. P. \& Zhu, C. 2007 Transport governs flow-enhanced cell tethering through L-selectin at threshold shear. Biophys. J. 92, 330-342. (doi:10.1529/biophysj.106.090969)

Zhao, Y. H., Chien, S. \& Weinbaum, S. 2001 Dynamic contact forces on leukocyte microvilli and their penetration of the endothelial glycocalyx. Biophys. J. 80, 1124-1140.

Zhu, C. 2000 Kinetics and mechanics of cell adhesion. J. Biomech. 33, 23-33. (doi:10.1016/S00219290(99)00163-3) 